\title{
دراسة معوقات تطبيق الادارة الاكترونية في مؤسسات التعليم العالي
}

\author{
سميعة مهذي حسن 1 وضاح عامر حاتم ²، جاسم خلف3، كوثز هاشم4 4 \\ 1، 2مدرس، 3، 4 مدرس مساعد /المعهة التقتي -بعقوية \\ sam_saf61@yahoo.com \\ (الاستلام:-2015/8/19 ، القبول:- 2015/11/11)
}

الخلاصة: هدفت هذه الدراسة إلى الكثف عن مستوى ادراك العاملين في مؤسسات التعليم العالي والبحث العلمي لاهمية تطبيق الإدارة الالكترونية في هذه المؤسسات والمعوقات الني تعرقل تطبيقها (محافظة ديالى، حالة دراسية). تم استخدم

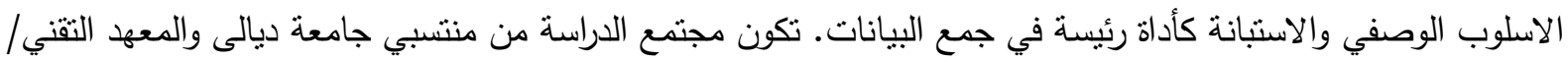
بعقوبة وطبقت الدراسة على عينة طبقية عشوائية وعددهم (111). بينت نتائج الدراسة ان اغلب العاملين يدركون جيدا اهية التحول نحو الادارة الالكترونية في مؤسسسات التعليم العالي كما ان اهم عائق يعرقل التحول هو "قلة الموارد المالية

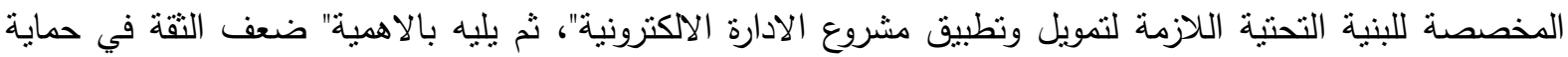

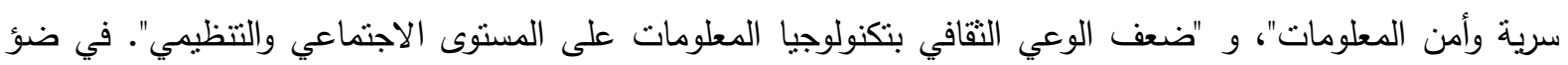

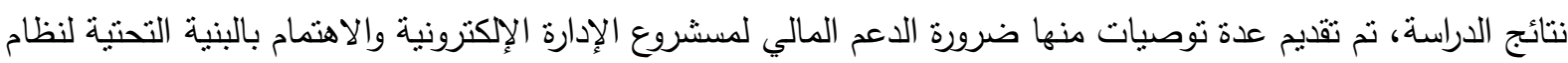
التعليم العالي لمواكبة النطور الذي يحصل في سباق التكنولوجيا. كذلك، توفير أساليب وإجراءات أمنية وقائية تضمن حماية البيانات من الاختراق.

الكلمات المفتاحية: الادارة الالكترونية، التعليم العالي والبحث العلمي، معوقات تطبيق الادارة الالكترونية

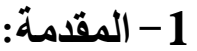

ان ظهور معايير وقيم حديثة للعمل الاداري شكل ضغطا على ادارة المؤسسات لتاخذ شكلا جديدا يوفر المناخ

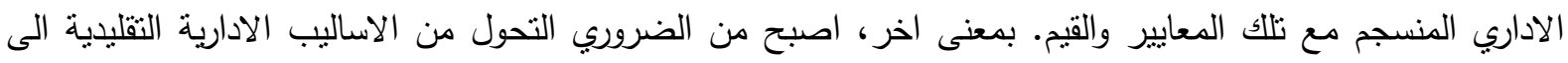
الاساليب الالكترونية(1). ذلك جعل تطوير النظم الادارية والتغيير التتظيمي من أهم سمات العصر الحديث لمواكبة المستجدات العالمية المنسارعة ومواجهة التحديات(2). وتبعا لذلك، فان جميع المؤسسات بحاجة الى تغيير اداراتها التقليدية وتنبي انظمة ادارية رقمية تتماشى مع سمات العصر الحالي (3).

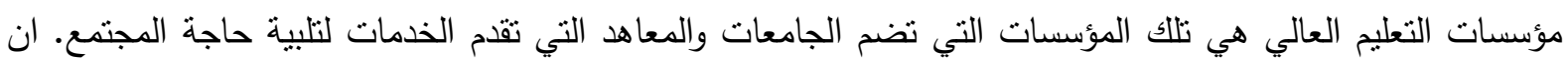

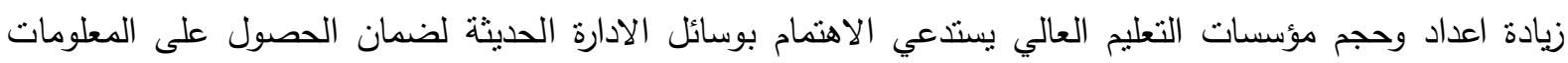

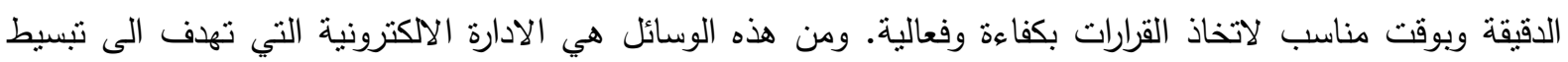

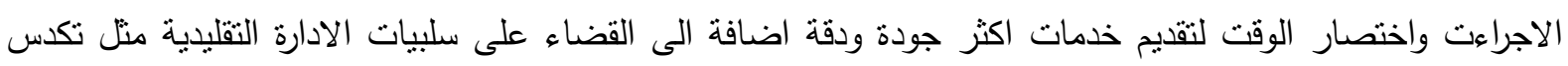
الاوراق(4) والروتين والبيروقراطية الادارية. من اهم عوامل نجاح ادارة المؤسسات، ومن ضمنها مؤسسات التعليم العالي

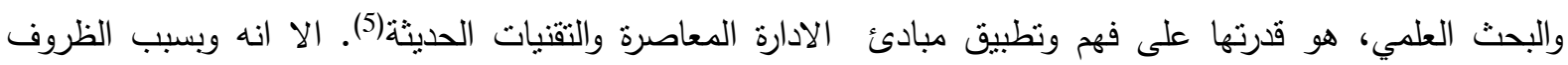
الامنية وعدم الاستقرار السياسي في العراق لاتوجد خطط استراتيجية واضحة المعالم في مجال تكنولوجيا المعلومات وعدم

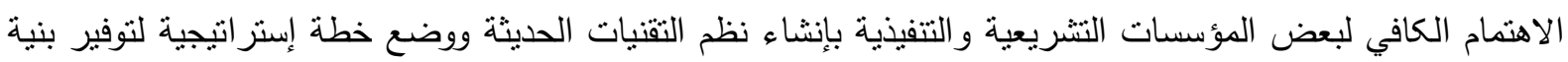


تحتية لنظم المعلومات والاتصالات لمواصلة التطور وبناء العراق الجديد(6). لذلك استمرت ادارة مؤسسات التعليم العالي تعمل وفق النظم الادارية التقليدية مما نتج وجود اعداد كبيرة من النسخ للكتب والاوامر الادارية محفوظة في اماكن مختلفة مما يؤدي الى زيادة في نفقات الارشفة واستهلاك الورق والوقت اضافة الى وجود الاخطاء والحاجة الى اعداد كبيرة من الموظفين عليه، اصبح من الضروري الانتقال من العمل الإداري التقليدي إلى تطبيق تقنيات المعلومات والاتصالات في

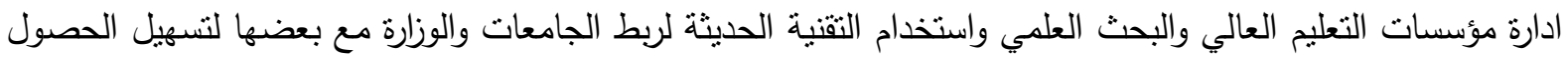
على البيانات والمعلومات وانجاز الأعمال وتقديم الخدمات للمستقيدين بكفاءة اعلى و بأقل تكلفة وأسرع وقت مدكن. الهدف من هذه الدراسة هو التعرف على مفهوم الادارة الالكترونية والقاء الضؤ على مدى ادرالك العاملين في مؤسسات التعليم العالي والبحث العلمي لاهمية التحول نحو الادارة الالكترونية، كذللك الكثف عن العوامل التي تحول دون تطبيقهاه في العراق من وجهة نظر العاملين في تلك المؤسسات. تاتي اهمية البحث من خلال اهمية تكييف مؤسسسات التعليم

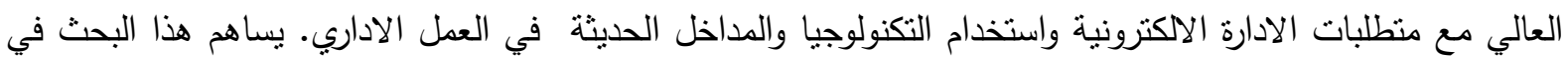
ابراز اهمية تطبيق الادارة الالكترونية في مؤسسات التعليم العالي والبحث العلمي للارتقاء بالاداء الجامعي ورفع كفاءة الادية الادارات الجامعية وتخفيف اعباء الادارة.

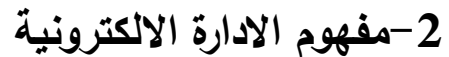

تم تداول تعاريف مختلفة في الادبيات للادارة الاككترونية، فقد عرفها ابو عاشور والنمري(7) بانها الاستثمار الإيجابي لتكنولوجيا المعلومات والاتصالات في جميع وظائف العملية الإدارية القائمة (التخطيط، والتنظيم، والتتفيذ، والرقابة، والمتابعة والتقويم)، وذلك بهدف تحسين أداءها وتعزيز مركزها التتافسي. اما السالمي(8)، فقد عرفها بانها عملية مكننة جميع مهام وأنشطة المؤسسات الإدارية بالاعنماد على تقنيات المعلومات الضرورية للوصول إلى تحقيق أهداف الإدارة الجديدة في ثقليل استخدام الورق وتبسيط الإجرءات والقضاء على الروتين. ويرى ياسبن (9) بأنها منظومة الأعمال

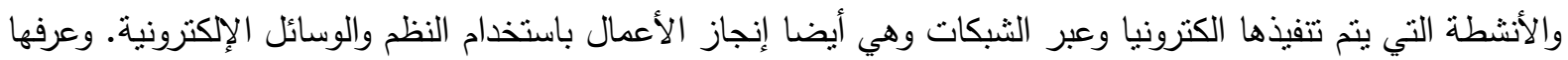
الموسى(10) انها اداء العمل الاداري باستخدام الحاسب الآلي في استقبال البيانات وتخزينها، والقيام بمعالجتها واستخراج النتائج المطلوبة بدقة وسرعة فائقة. في حين ان الفريح(11) عرفها بأنها أسلوب جديد للعمل الإداري باستخدام التقنيات الحديثة المتمثلة بالحاسب الالي والثبكة الدولية للمعلومات (الانترنيت) لتحقيق الكفاءة والفعالية في اداء العمل. وفي مجال تطبيق الادارة الاككترونية بالجامعات، عرفها الاغا واخرون(12) بانها استخدام الجامعات التقنيات والاتصالات والمعلومات

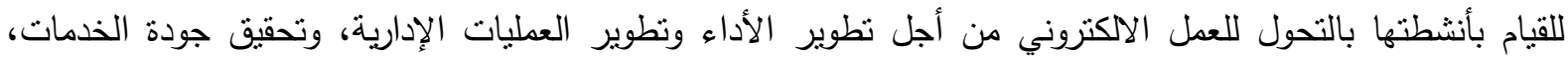

$$
\text { وتحقيق الأهداف بأقل وقت وجهد وتكلفة. }
$$

ومن خلال الدراسات اعلاه يمكن ان يعرف الباحثون الادارة الالكترونية في مؤسسات التعليم العالي بانها انتقال العمل الاداري التقليدي في ادارة مؤسسات التعليم العالي الى العمل الاداري الالكتروني من خلال استخدام تقنيات

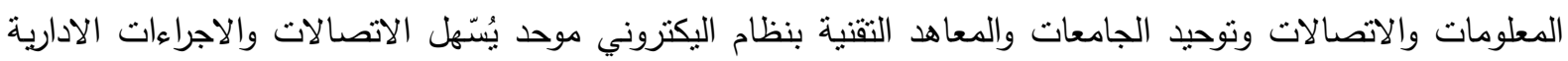

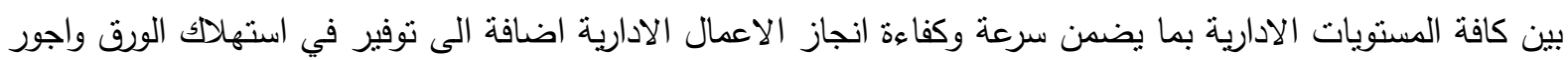
النقل وغيرها.

ان الإدارة بمفهومها التقليدي لم تعد قادرة على تحقيق أهداف المؤسسات في ظل النطور التقني والمعرفي في نظم المعلومات، حيث انها تتظيمات جامدة بمكان وزمان محدد وفقا لمواعيد العمل الرسمية اضافة الى كثافة استعمال

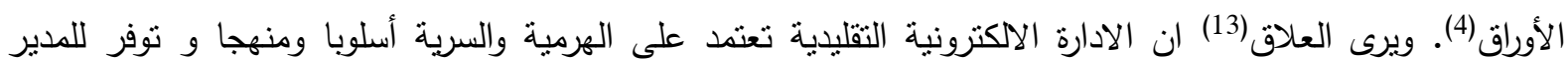

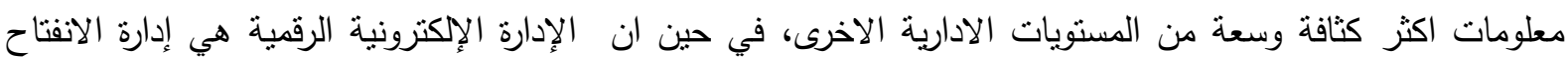

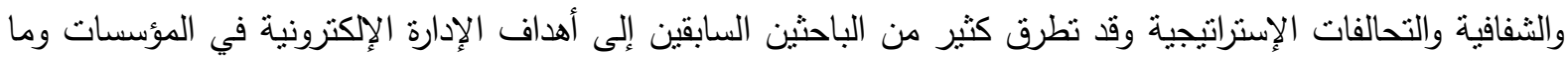


يمكن ان تحققه في تحسين مستوى الاداء الاداري والتتظيمي. فقد اثار النجار (14) الى ان الادارة الآكترونية تقلل التعقيدات الادارية من خلال الحد من البيروقراطية في اسلوب الادارة وبذلك ينطور مستوى الخدمات عن طريق تجاوز الاخطاء التي تحدث في الادارة التقليدية. وذكر نجم(15) بإن الإدارة الالكترونية تعمل على تحقيق المرونة الادونة الإدارية في

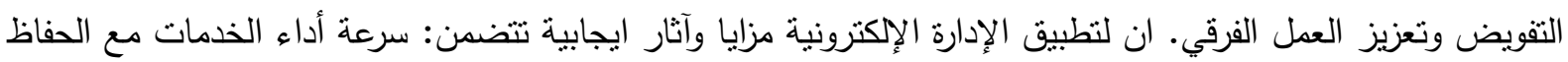

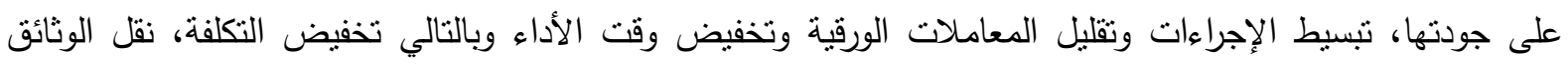

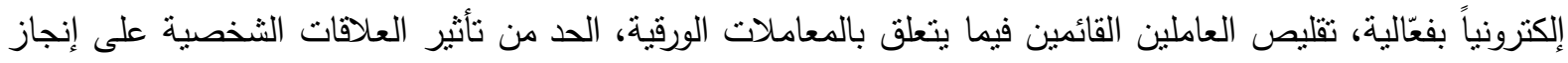

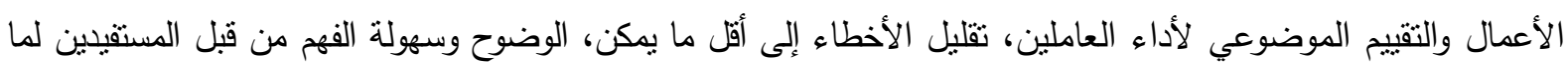

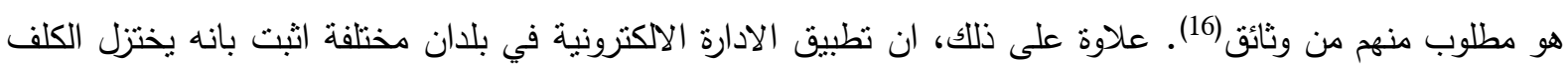

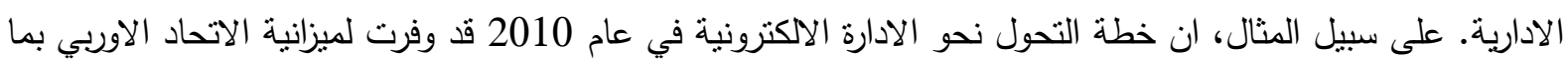
يعادل 50 مليون يورو سنويا(17).

\section{3- معوقات تطبيق الادارة الاككترونية}

تتبهت كثير من البلدان العربية مؤخراً إلى اهمية الدخول في عالم الاقتصاد المعلوماتي فبدأت بوضع سياسات لتحقيق هذا الهدف، إلا أن كثير من الدول العربية (ومنها العراق) لاتزال تقتقر لوجود سياسات واستراتيجيات واضحة في مجال الاتصال وتبادل المعلومات. ومن اهم الاسباب هو ضعف الإن الوعي الاجتماعي والثقافي بمفهوم الادارة الالكترونية

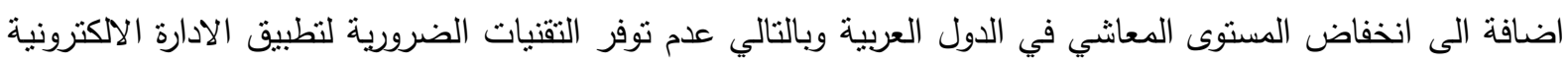

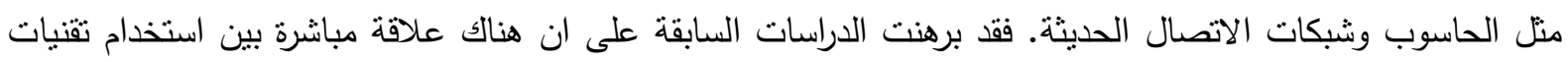
الحاسوب والاتصالات الحديثة وبين مؤشر التتمية البشرية (HDI)(18)، وهو مؤشر ابتكرته هيئة الأمم المتحدة يشير إلى فئي مستوى رفاهية الثعوب في العالم. ويتعلق هذا المؤشر بقياس منوسط العمر المنوقع للمواطن ومستوى التعليم والأمية

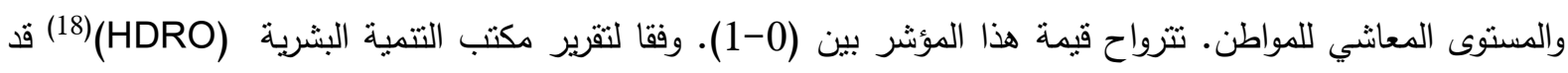

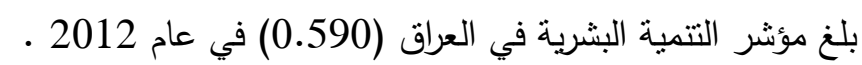
طبقا لاحصائيات الاتحاد الدولي للاتصالات (ITU)(19)، ان العلاقة بين مؤنشر التتمية البشرية وعدد الحواسيب الثخصية لكل 100 فرد يمكن توضيحها بالثكل رقم (1). كما ان عدد الحواسيب الثخصية لكل 100 شخص في العراق قد بلغ 0.757429 وذلك في عام 2002. ومن الثكل رقم (1) يتضح ان الفجوة في عدد الحواسيب الشخصية

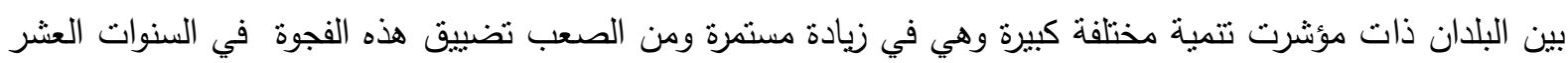

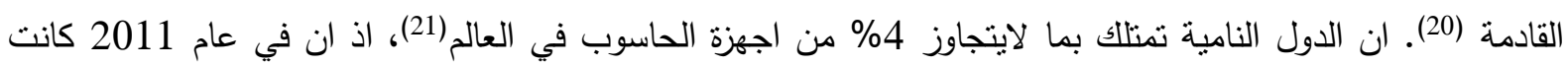
نسبة امتلاك الحاسب الثخصي لاتجاوز (17.2\%) كما ورد في دراسة قامت بها منظمة اليونسكو (22).

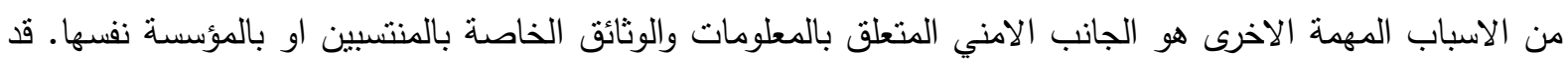
بينت دراسة الاسدي(6) ضعف الاجراءات الفنبة والادارية للمؤسسات لغرض حماية البيانات وضمان الامن والان والسرية والخصوصية.

\section{4-منهجية البحث}

استخدم الباحثون المنهج الوصفي التحليلي لغرض التعرف على مفهوم الادارة الالكترونية والعوامل التي تعيق

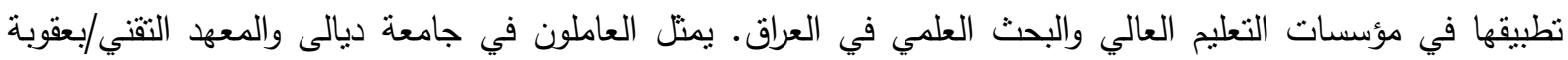
مجتمع البحث. تم اعتماد الاستنيان كاداة لجمع البيانات وذلك لامكانية تطبيقه على نطاق واسع أو على عينات كبيرة

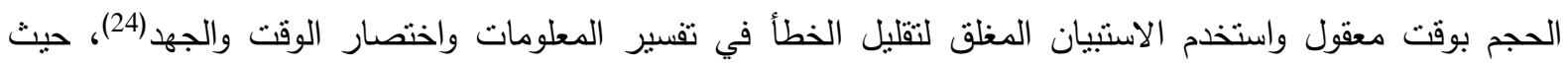
تضمن الاستبيان عدة اسئلة والملطلوب من المستجيب أن يختار الاجابة المناسبة من بين عدة اجابات. 
بعد ان تم وضع استمارة الاستبيان في صورتها الأولية نم عرض الاستمارة على احد عثر من أساتذة الجامعات في تخصصات متعددة ممن يعملون في جامعة ديالى والمعهد التقني/بعقوبة، ولهم خبرة لاتقل عن 15 سنة لتقويم الاستبيان (تحكيمه) على أساس القواعد العلمية في ضوء أهداف البحث، اذ نم التداول والتحاور واستطلاع رأيهم حول مسودة نموذج وله الاستبيان المطروحة من حيث الصيغة والأسلوب وتدعيمه بأسئلة إضافية ناجمة عن خبرتهم. بعد إكمال المقابلات الثخصية ومناقثة الأسئلة الواردة في استمارة الاستبيان المقترحة بشكلها الأولي نم جمع البيانات وجدولتها كما مبين في بئه

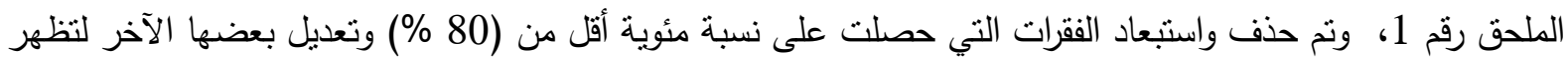
بصورتها النهائية، بذلك نم التحقق من صدق المحتوى (Content Validity) والذي هو صفئ أساسية من صفات الاختبار الجيد.

تتضمن استمارة الاستبيان قسمين، الأول عبارة عن معلومات شخصية عن عينة البحث اما القسم الثاني فيتضمن محورين

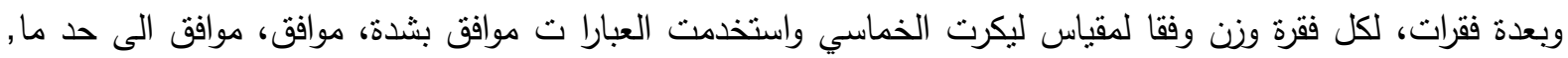
غير موافق، غير موافق على الإطلاق وتمنل 5، 4، 3، 2، 1 على التوالي. ونم توزيع 150 استمارة على عينة طبقية

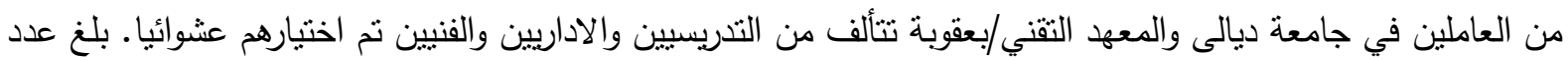

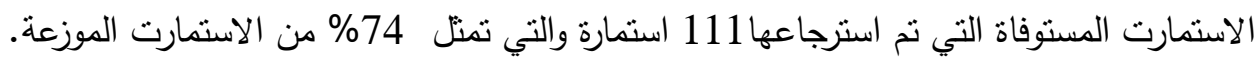

نم استخدام المعالجات الاحصائية التالية: معامل الفا كرونباخ لحساب معامل الثبات لمجتمع الدراسة الاسنطلاعية، التوزيعات التكرارية والنسب المئوية، المتوسطات الحسابية والانحرافات المعيارية،الوسط الحسابي المرجح، معامل ارتباط الهابه بيرسون لقياس ثبات الاتساق الداخلي للاستبانة، اختبار تحليل التباين الاحادي انوفا ANOVA وذلك باستخدام الحقيبة

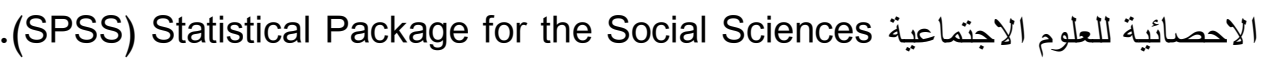

5- 5 النتائج والمناقشة 5-1-5 الصدق والثبات

جرى التحقق من صدق الاتساق الداخلي للاستبانة بتطبيق الإستبانة على عينة استطلاعية مكونة من (32) عاملاً من العاملين في جامعة ديالى والمعهد التقني/بعقوبة، و تم احتساب معامل ارتباط بيرسون بين كل فقرة من فقرات الاستبانة والدرجة الكلية للاستبانة، وذلك باستخدام البرنامج الإحصائي (SPSS)، وقد تبين ان جميع الفقرات ترتبط ارتباطاً معنويا

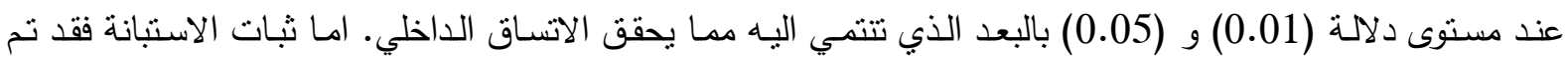
استخدام طريقة التجزئة النصفية حيث احتسبت درجة النصف الأول لكل بعد من أبعاد الاستبانة وكذلك درجة النصف الثاني من الدرجات وذلك بحساب معامل ألفا كرونباخ، وقد تجاورز 0.7 مما يجعل الاسنبانة مقبولة الثبات. 2-5 2-5 20نة البحث

• يتضمن هذا المحور أسئلة عامة تهدف الى جمع البيانات والمعلومات عن أفراد العينة. وقد تمثلت الأسئلة حول المكان الذي يعمل فيه الفرد، الثهادة الحاصل عليها، الاختصاص الذي درسه وكذلك الدرجة الوظيفية، إضافة الى عدد سنوات الخدمة, كما مبين في الجدول رقم (1).

تضمنت العينة اعلى نسبة لحملة شهادة الباكالوريوس (52.3\%)، كما مبين في الجدول رقم (1). اما اللقب العلمي فكان حوالي تلث العينة (31.5\%) اداريون وخمس العينة (21.6\%) بلقب مدرس ونفس النسبة يحملون لقب مدرس مساعد. بالنسبة لسنوات الخبرة فان اغلب افراد العينة يمتلكون خبرة بين 11-25 سنة. اكثر من نصف العينة (51.4\%) يعملون في الجامعة، وحوالي نصف العينة (42.7\%) بشغلون وظيفة تدريسي. 
أظهرت نتائج الاستبيان أن معظم الذين شملهم الاستنيان يتفقون على ان اهميـة تطبيق الادارة الاككترونية تاتي بالدرجـة

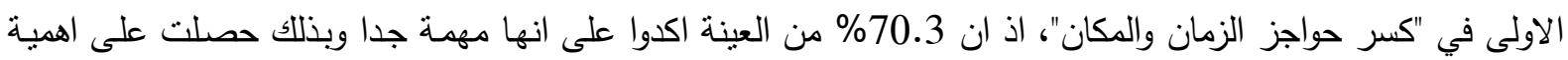

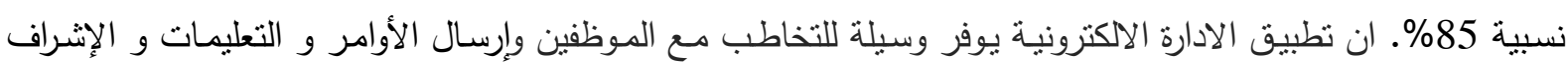
على الأداء و إقامة الندوات و المؤتمرات من خلاد "الفيديو كونفرانس" و من خلال الثبكة الالكترونية للإدارة، وبذلك يتم اختصـار الوقت وتجاوز المكان. كذلك انها تلغي تاثير عامل الزمن من ناحية وقت العمل وبغض النظر عن انت ان يكون

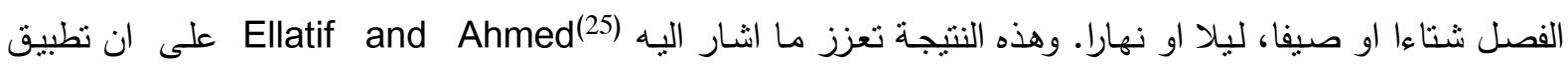
الادارة الالكترونية يؤدي الى اختصار الكلفة والزمن.

اكد 65.8\% من افراد العينة ان "سرعة وصول القرارات والتعاميم" من الامور التي تهدف اليها الادارة الالكترونية وبذلك

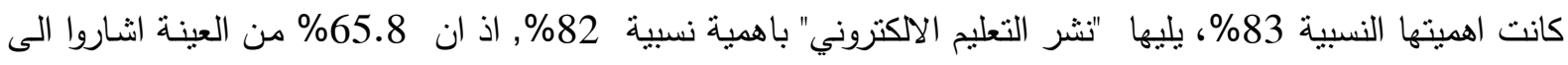
انها مهمة جدا. كما ايد معظم افراد العينة على ان تطبيق الادارة الاككترونية "يوفر تجميع البيانات من مصادر ها الأصلية

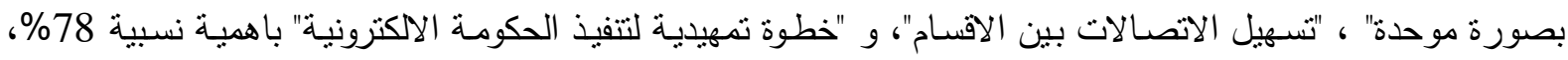
\%77، 76 \% الذين اقترحوا ونفذوا تصميما لنموذج لـلادارة الاككترونيـة لمرافق المعهد التقني في اربيل واكدوا ان هذا النموذج وفر وقتـا وكلفة اضـافة الى سـهولة الحصول على البيانات والمعلومات.

يلاحظ من الجدول رقم (2) ان جميع قيم مربع كاي (X2) دالة معنويا عند مسنوى دلالة (0.00-0.05) مما يؤكد انه

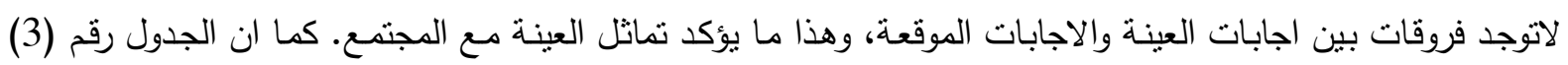
يبين انه لاتوجد فروقات ذات دلالة احصائية عند اي مستوى دلالة بين استجابات افراد العينة تبعا للعمل الحالي، المؤهل

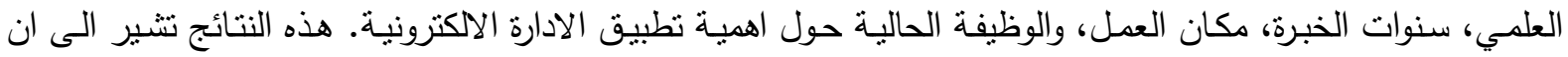

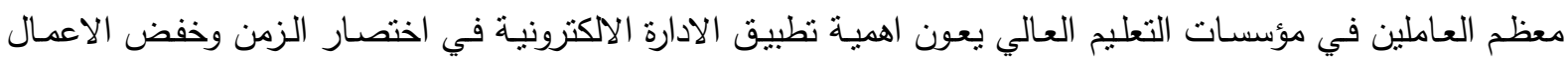

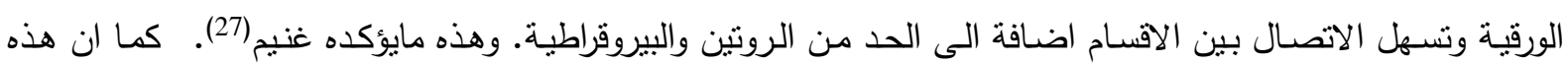
النتائج تتفق الى حد ما مع رحو واخرون (28)، اذ نوصلوا الى ان الاغلبية يؤيدون تطبيق الادارة الآكترونية في مؤسسات التعليم العالي.

\section{5-4 معوقات تطبيق الادارة الاكترونية}

ان وضع استراتيجية متكاملة للانتقال إلى نمط الإدارة الاككترونية لا يعني أنّ الطريق ممهدة لتطبيق و تتفيذ هذه

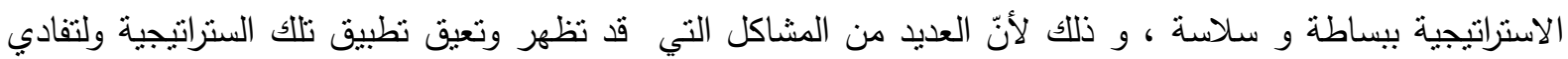
هذه المشاكل أو وضع الحلول المناسبة لها، من الضروري معرفة و ادراك كافة العناصر و المتغيرات التي يمكن أن نطرأ وتتخيصها في البيئة التي يراد نطبيق الادارة الالكترونية فيها. وعند الاستفسار عن هذه العوائق من وجهة نظر العاملين

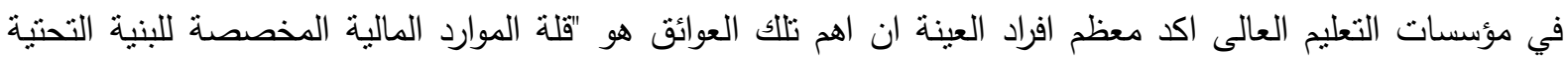

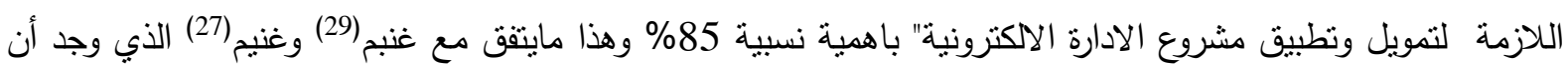

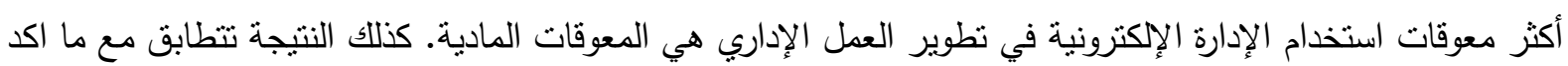

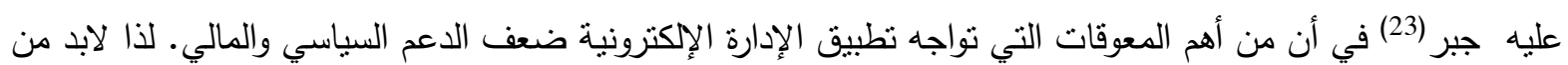

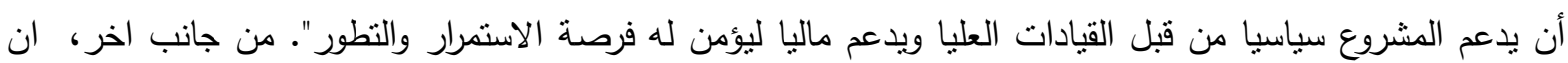


النتيجة لاتتفق مع ما توصل اليه Gilaninia et al.(30) في ايران، اذ وجدوا ان المعوقات المالية ناني في المرتبة الرابعة في الاهمية. والسبب يمكن ان يعزى الى اختلاف الظروف الاقتصادية بين البلدين؛ ايران والعراق.

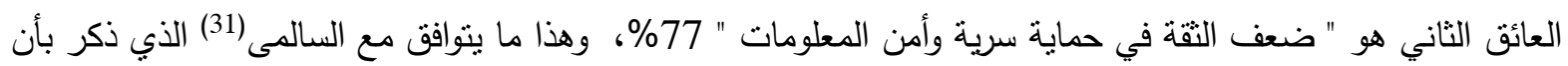
التطورات المتسارعة في العالم ادى بنفس الوقت الى نظوير الإمكانات والتقنيات الرامية إلى خرق منظومات الحواسيب

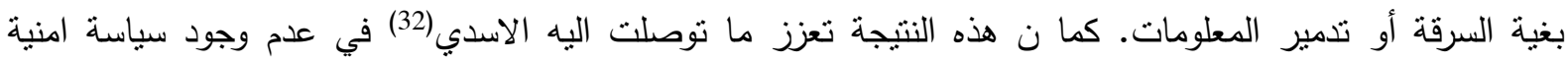
واضحة لحماية البيانات وضمان المحافظة على الامن والسرية والخصوصية في العراق. كما ان الباحثة اشارت الى لى اندان مصدر الخطورة لاياتي من تطبيق الادارة الالكترونية، بل في عدم اتخاذ الاحتياطات الامنية مما يشجع على التجسس لهابل

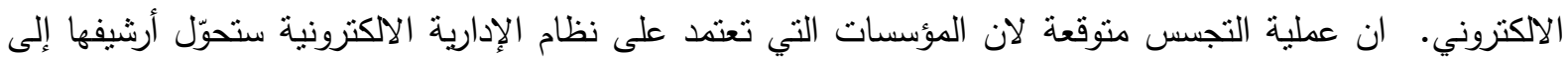
أرشيف الكتروني وهو ما يعرّضه لدخاطر التجسس على هذه الوثائق و كثفها و نقلها او إتلافها لذلك فهناك مخاطر كبيرة من الناحية الأمنية على معلومات و وثنائق و أرشيف الإدارة سواء المتعلقة بالأشخاص أو الثركات أو الإدارات. اما العائق الثالث في الاهمية من وجهة نظر العينة فهو "ضعف الوعي التقافي بتكنولوجيا المعلومات على المستوى الاجتماعي و التتظيمي"، (69\%). هذه النتيجة تتفق مع نتائج دراسة العواملة(33) حول المعوقات التي قد تعرقل التحول نحو الحكومة الإكترونية والتي تضمنت ضعف الوعي الاجتماعي، ونقص التمويل والكفاءات البشرية، المعلومات

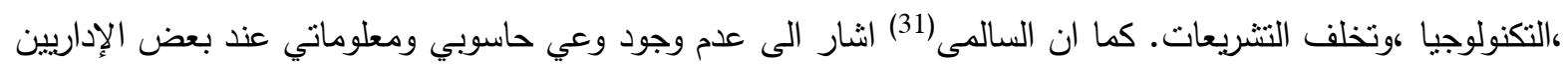

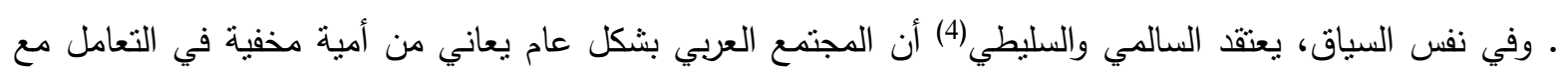

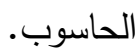

اما اقل المعوقات اهمية فنتشل" ضعف البنية التحتية للمؤسسات الجامعية وعدم جاهزيتها لإستقبال منل هذه التقنية " و " ندرة وجود مواصفات ومعايير موحدة للأجهزة المستخدمة حتى داخل الجامعة الواحدة "، فقد اكد معظم افراد العينة ان هذين العائقين غير مهمين اذ بلغت الاهمية النسبية لكل منهما (35\%). هذه النتيجة تتاقض ما اشتار اليه كلا من جبر (23) و السالمي(31) بان عدم وجود بنية تحتية لنظم المعلومات والاتصالات او وضعف كفاءتها التشغيلية واختلاف القياس لهاس والمواصفات بالأجهزة المستخدمة داخل المكتب الواحد من أهم المعوقات التي تواجه تحول المنظمات نحو البيئة

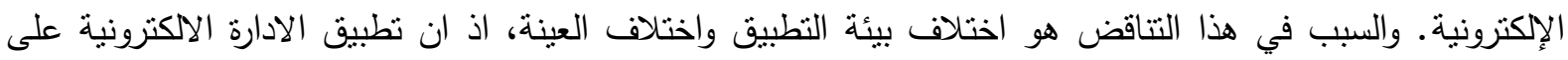

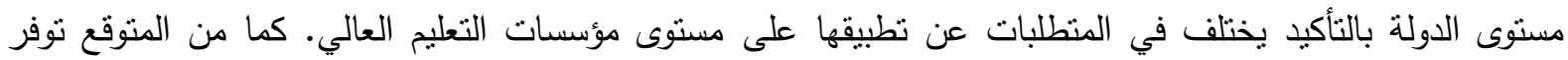

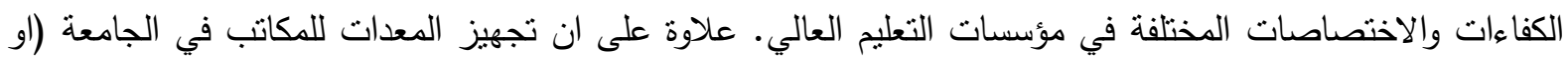
المعهد) مركزي، لذا يتوقع ان تكون مواصفات ومعايير موحدة للأجهزة الواحدة اما بقية المعوقات فتترواح الهميتها النسبية بين (0.63) - 0.45).

من الجدول رقم (4) يمكن ملاحظة ان تطابق التكرارين الملاحظ والمنوقع ، اذ ان قيم مربع كاي( (X) ) دالة معنويا عند مستوى الدلالة (0.01) عدا الفقرة الاولى " انعدام التخطيط والتتسيق على مستوى الإدارة العليا لبرامج الإدارة الإلكترونية" ، اذ ان قيمة مربع كا (X) غير دالة معنوبا عتد اي مستوى دلالة. قد يكون السبب هو ان ثقيبم سياسات الادارة العليا

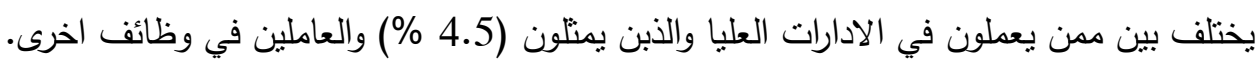
من جانب اخر ، يوضح جدول (5) بانه لاتوجد فروقات ذات دلالة احصائية عند اي مستوى دلالة بين استجابات افراد العينة تبعا للعمل الحالي، المؤهل العلمي، سنوات الخبرة، مكان العمل، والوظيفة الحالية حول معوقات تطبيق الادارة الالكترونية. 
كثفت الدراسة عن اهم المعوقات التي قد تعرقل التحول نحو الادارة الالكترونية في مؤسسسات التعليم العالي، كان اهمها: "قلة الموارد المالية المخصصة للبنية التحتية اللازمة لتمويل وتطبيق مشروع الادارة الالكترونية"، " ضعف التقة في حماية سرية وأمن المعلومات "، و "ضعف الوعي الثقافي بتكنولوجيا المعلومات على المستوى الاجتماعي و التنظيمي". على صعيد اخر، بينت الدراسة ان اعلب العاملين في مؤسسة التعليم العالي يعون اهمية تطبيق الادارة الالكترونية، رغم الحاجة الى تتمية الوعي التقافي بتكنولوجيا المعلومات والاتصالات وتدريب الموظفين على استخدام النظام الالكتروني والاعتماد

في ضؤ نتائج الدراسة يوصي الباحثان على ضرورة الدعم المالي لمسشروع الإدارة الإكترونية والاهتمام بالبنية التحتية

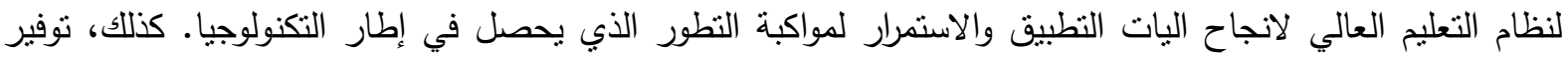
أساليب وإجراءات أمنية وقائية تضمن حماية البيانات من الاختراق.

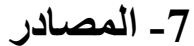

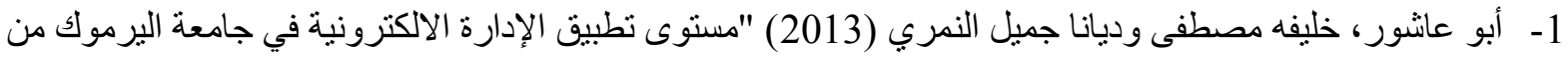

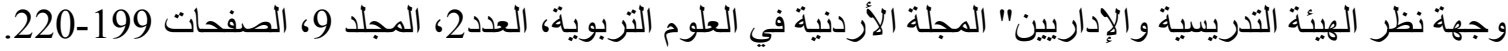

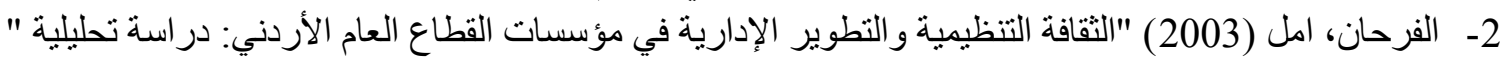
المجلة الاردنية للعلوم التطبيقية، العدد الاول، المجلد 6. 3- ماهر ، أحمد (2007) " تطوير المنظمات: الدليل العلمي لإعادة الهيكلة والتميز الإداري و إدارة التغير" الاسكندرية،

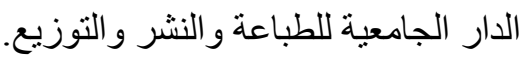
4- السالمي، علاء عبد الرزاق و السليطي، خالد ابراهيم (2009) "الادارة الالكترونية" الطبعة الثانية، عمان، دار و وائل للنشر.

5- Pougatchev, V. and Kulkarni, A. B. (2011) "Technical Aspects of the Integrated Management Information system for Educational Institutions" International Journal of Systems applications, Engineering \& Development, Issue 4, Volume 5, pp 570-583. 6- الاسدي، عدي غني (2011) "الاستثمار في تقنيات المعلومات ودوره في التنمية الاقتصادية/العراق نموذجا" مجلة

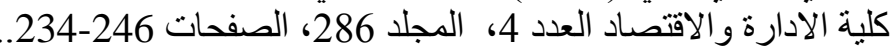

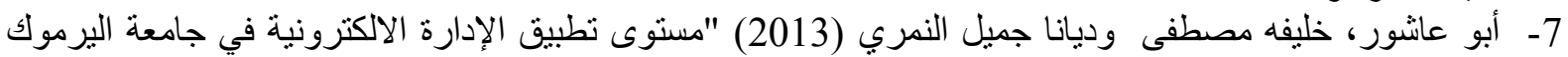

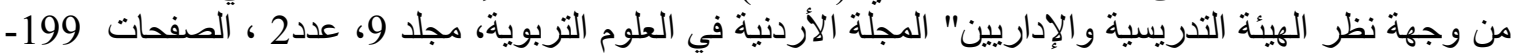

8- السالمي, علاء عبد الرزاق ( 1999 ) ) "نظام المعلومات و الذكاء الاصطناعي" عمان, دار المناهج للنشر و التوزيع الإنيع 9- ياسين، سعد غالب (2005) "الإدارة الإلكترونية وآفاق تطبيقاتها العربية " الرياض، معهذ الإدارة العامة، مركز

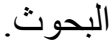
10ـ الموسى، عبد الله ( 2002 ) "استخدام الحاسب الآلي في التعليم" الرياض، مطابع جامعة الإمام محمد بن سعود

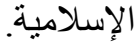

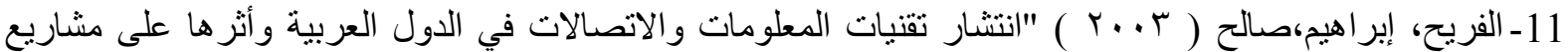

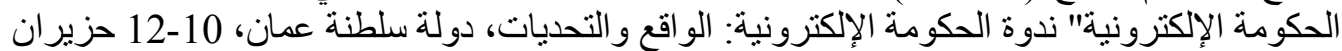

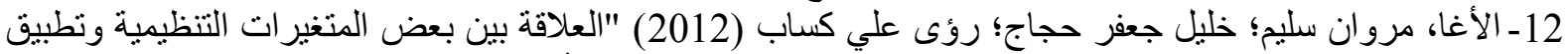

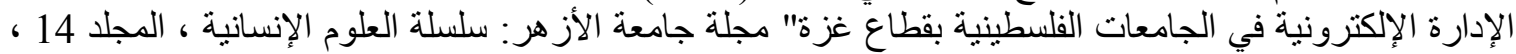

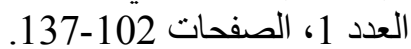
13ـ العلاق، بشير عباس (2005) "الإدارة الرقمية: المجالات والتطبيق" ابو ظبي/الإمارات العربية ، مركز الإمارات

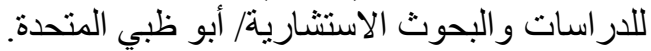

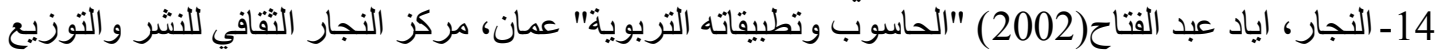

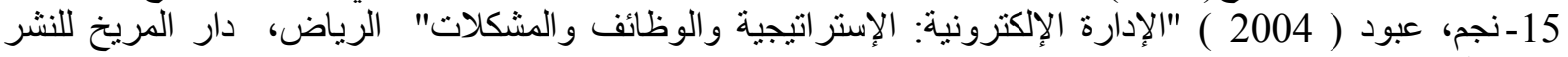
و التوزيع. 
16- Munoz-Canavate, A. and Hípola, P. (2011) "Electronic administration in Spain: From its beginnings to the present" Government Information Quarterly, beginnings to the present" Government Information Quarterly, Volume 28, Issue 1, pp74-90.

17- Human Development Report (2013) "The Rise of the South: Human Progress in a Diverse World" United Nations Development Programme, pp 144-147, Retrieved 21 March 2015 from http://hdr.undp.org/en/2013-report

18- International Telecommunication Union World Telecommunication Development (2009), ICT Indicators Database, Retrieved on 21 March 2015 from http://www.econstats.com/wdi/wdiv_597.htm

19-.ITU Statistics online database http://www.itu.int/ITU-D/ict/ and World Telecommunication / ICT Indicators Database 2009, Retrieved 22 May 2015 from http://www.econstats.com/wdi/wdiv_597.htm

20- Blake, A. and Garzon, M. Q. (2010) "ICT for Development: sustainable technologysupported participatory development for poverty alleviation in the context of digital divides" Centre for Development Research/Zentrum für Entwicklungsforschung, $\begin{array}{llllll}\text { University of } & \text { Bonn } & \text { Retrieved } & \text { Jan. }\end{array}$ from: https://researchspace.auckland.ac.nz/docs/uoa-docs/rights.htm

21-Harris, R. W. (2004) "Information and communication technologies for poverty alleviation" The United Nations Development Programme's Asia-Pacific Development Information Programme, Retrieved $20 \quad 2015$ from http://www.apdip.net/publications/iespprimers/eprimer-pov.pdf

22- United Nations Educational Scientific and Cultural Organization (UNESCO) (2004) "Iraq Education in Transition: Needs and Challenges,"Paris, France, Retrieved 20 Jan. 2015 from http://www.unesco.org/education/iraq/na_13jan2005.pdf.

23-جبر، محمد صدام (2002) "الموجة الالكترونية القادمة: الحكومة الالكترونية" مجلة الإداري، العدد 91، الصفحات176-200.

24- الجرجاوي، زياد بن علي بن محمود (2010) "القواعد المنهجية لبناء الاستبيان" الطبعة الثانية، فلسطين- غـز ة، مطبعة أبناء الجراء زبراء

25- Ellatif, H. A. A. and Ahmed, S. A. (2013) "E-Management: Configuration, Functions and Role in Improving Performance of Arab Institutions and Organization" International Journal of Computer Applications, Volume 80 - Issue.6, pp 0975 - 8887.

26- Ismaeel, A. G. and Mikhai, D. Y. (2011) "Design of Locally E-management System for Technical Education Foundation- Erbil" Computer Science and Telecommunications, Volume. 1, Issue 1, PP 21-28

27- غنيم، احمد محمد (2004) "الادارة الالكترونية: افاق الحاضر وتطلعات المستقبل" مصر - المنصورة، المكتبة العصرية

28-Raho, G. I., Muzhir Shaban Al-Ani , Khattab M. Ali Al-Heeti (2015) "E-University Environment Based on E-management”, International Journal of Computational Engineering Research, Volume, 05 , Issue, 04 PP 29-34

$$
\begin{aligned}
& \text { 29- غنيم، احمد علي (2006) "دور الإدارة الالكترونية في تطوير العلم الإداري ومعوقات استخدامها في مدارس التعليم } \\
& \text { العام في المدينة المنورة" المجلة التربوية، العدد 81، مجلد 21، الصارة الصفحات 143-220. }
\end{aligned}
$$

30-Gilaninia, S., Seyyed Javad Mousavian, Soheil Ghorbani Ghavidel Boeini, Alireza

Najafpour, Mohammad Najibzadeh, Hatef Esmaeili, Mohammad Babaei, Fatemeh Zadbagher Seighalani (2011) "Challenges Application of E-Commerce in Iran" Interdisciplinary Journal of. Contemporary Research in Business Vol. 3, No 8, PP 479507.

31- السالمى، علاء عبد الرزاق محمد و السالمى، حسين علاء عبد الرزاق (2009) "شبكات الإدارة الالكترونية" الطبعة

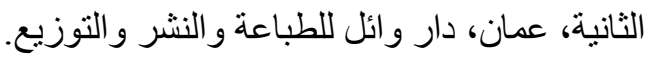


32- الاسدي، افنان عبد علي (2009) "الادارة الالكترونية بين النظرية ومتطلبات النطبيق في بيئة منظمات الاعمال

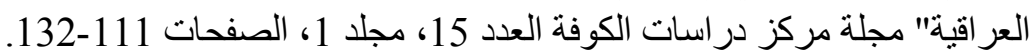

33-العو املة، نائل عبد الحافظ (2003) " الحكومة الإلكترونية ومستقبل الإدارة العامة، در اسة استطلاعيّة للقطاع العام في

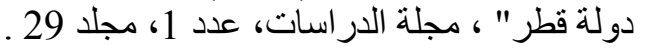

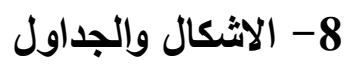

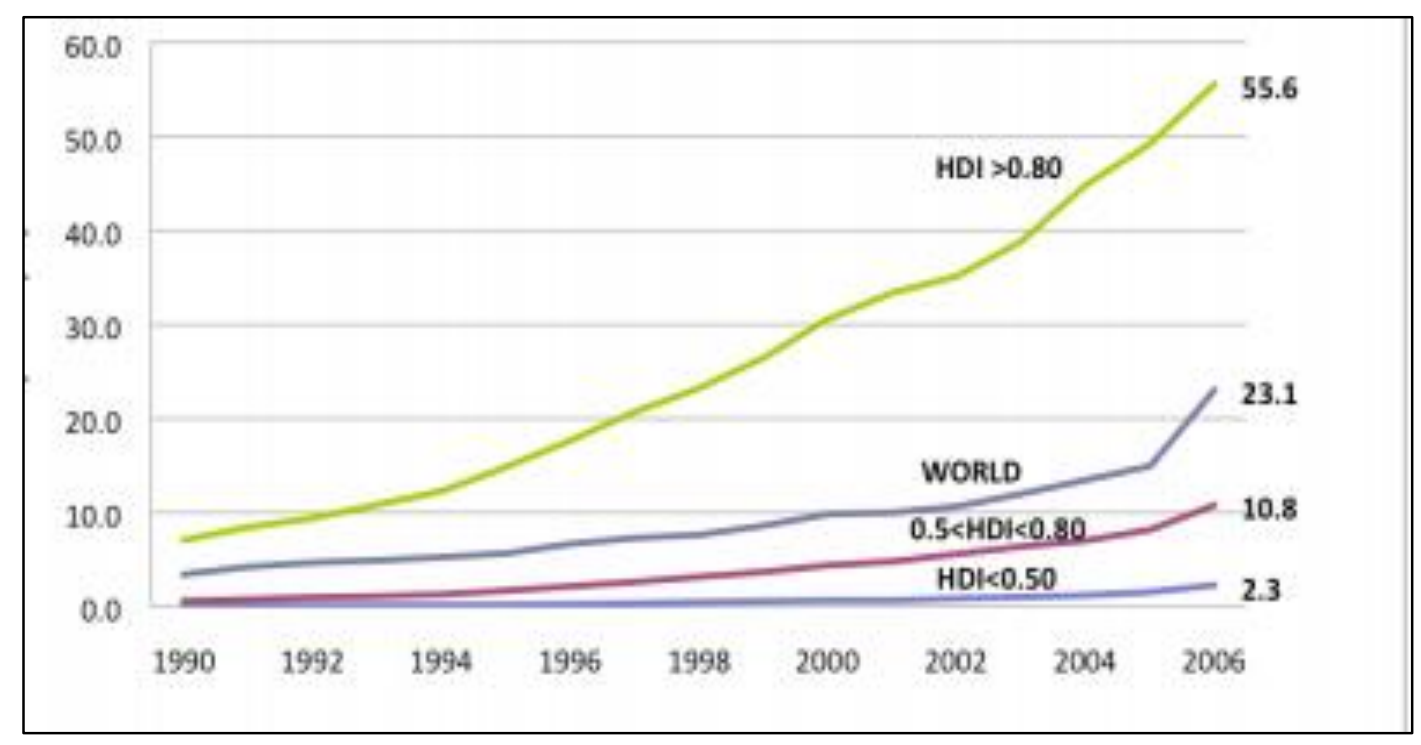

شكل رقم (1): العلاقة بين عدد الحواسيب الشخصية لكل 100 مواطن و مؤشر التنمية البشرية (ITU)،2009)

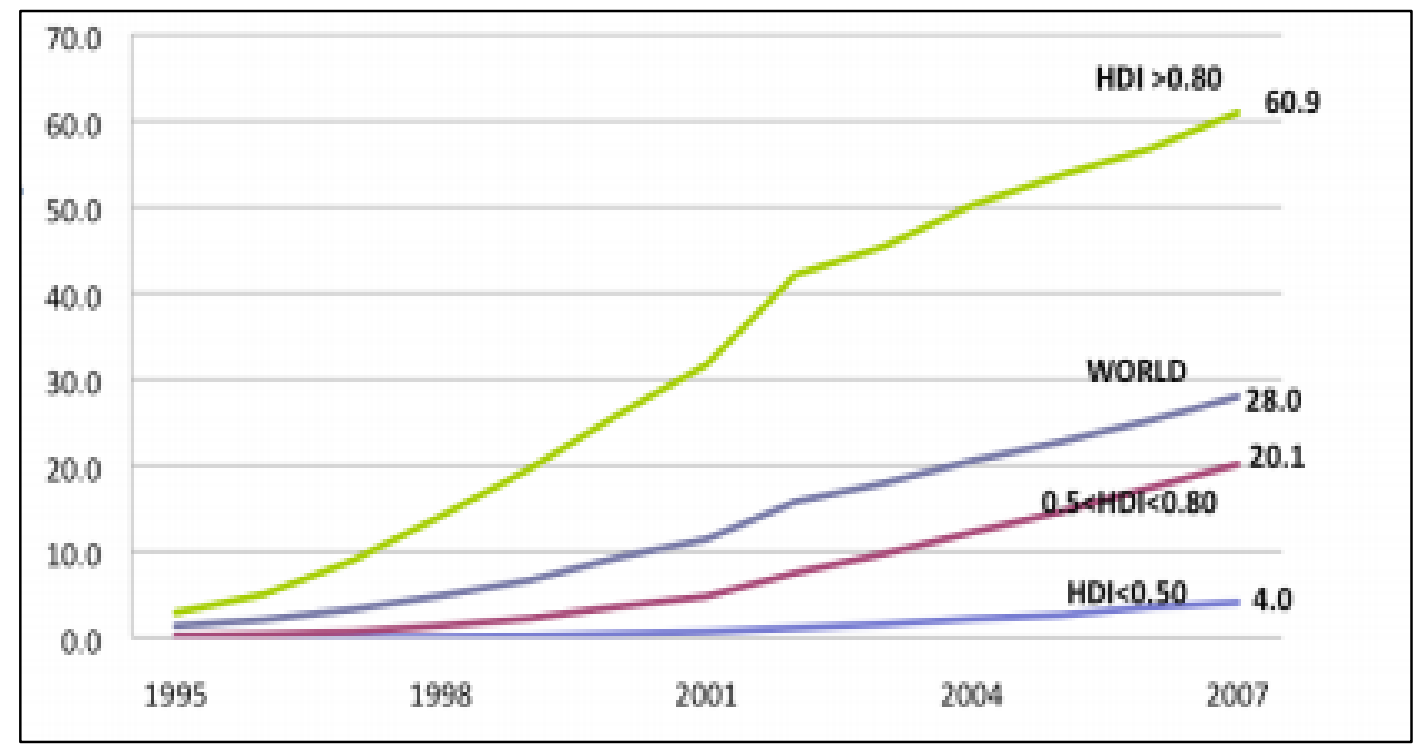

شكل رقم (2): العلاقة بين عدد عدد مستخدمي شبكات الانترنيت و مؤشر التنمية البشرية (ITU)،209) 


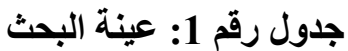

\begin{tabular}{|c|c|c|c|}
\hline النسبة التر اكمية & النسبة المئوية & السمات الشخصية & المحور العام \\
\hline 10.8 & 10.8 & دكتور اة & \\
\hline 27.9 & 17.1 & ماجستير & \\
\hline 80.2 & 52.3 & بكالوريوس & المؤ هل العلمي \\
\hline 100 & 19.8 & دبلوم & \\
\hline 1.8 & 1.8 & استاذ & \\
\hline 7.3 & 5.5 & استاذ مساعد & \\
\hline 28.9 & 21.6 & مدرس & \\
\hline 50.5 & 21.6 & مدرس مساعد & اللقب العلمي \\
\hline 68.5 & 18 & فني & \\
\hline 100 & 31.5 & اداري & \\
\hline 9.9 & 9.9 & اقل من 5 سنوات & \\
\hline 24.3 & 14.4 & $5-10$ & \\
\hline 45.9 & 21.6 & $11-15$ & سنو ات الخبرة \\
\hline 74.7 & 28.8 & $16-20$ & \\
\hline 95.4 & 20.7 & $21-25$ & \\
\hline 100 & 4.6 & اكثر من 25 & \\
\hline 51.4 & 51.4 & الجامعة & مكان العمل \\
\hline 100 & 48.6 & المعهد & \\
\hline 0.9 & 0.9 & عميد & \\
\hline 4.5 & 3.6 & م. عميد & \\
\hline 15.3 & 10.8 & ر. قسم علمي & \\
\hline 58.5 & 43.2 & تدريسي & الوظيفة الحالية \\
\hline 77.5 & 19 & مسؤول قسم اداري & \\
\hline 100 & 22.5 & اداري & \\
\hline
\end{tabular}




\section{جدول رقم 2: اهمية تطبيق الادارة الاكترونية}

\begin{tabular}{|c|c|c|c|c|c|c|c|c|c|}
\hline الفا & $x^{2}$ & الاهمية & 5 & للتكرار & المئوية & النسبة 2 & 1 & 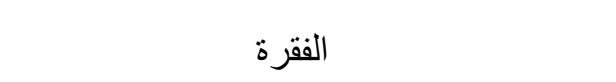 & ت \\
\hline 0.00 & 60.32 & 0.83 & 65.8 & 27.9 & 6.3 & 0.00 & 0.00 & سر عة وصول القرارات والتعاميم & 1 \\
\hline 0.00 & 18.21 & 0.77 & 40.5 & 45.1 & 14.4 & 0.00 & 0.00 & تسعيل الاتصالات بين الاقسام & 2 \\
\hline 0.00 & 18.24 & 0.85 & 70.3 & 29.7 & 0.00 & 0.00 & 0.00 & كسر حواجز الزمان والمكان & 3 \\
\hline 0.00 & 55.30 & 0.67 & 15.3 & 41.5 & 42.3 & 0.90 & 0.00 & اتخاذ القرارات بسر عة وفعالية & 4 \\
\hline 0.00 & 78.58 & 0.62 & 5.4 & 36.0 & 54.1 & 4.50 & 0.00 & القضاء على البيروقر اطية و الروتين & 5 \\
\hline 0.00 & 158.58 & 0.58 & 3.6 & 18.0 & 75.7 & 2.70 & 0.00 & اكثراحة مزيد من الوقت للعاملين لاداء مسؤليات & 6 \\
\hline 0.00 & 50.91 & 0.56 & 1.8 & 29.7 & 48.7 & 19.8 & 0.00 & زيادة النزاهة و الثفافية في العمل & 7 \\
\hline 0.00 & 20.10 & 0.71 & 20.7 & 53.2 & 26.1 & 0.00 & 0.00 & فورية البيانات و المعلوماتَ للمستفيدين بصورة & 8 \\
\hline 0.00 & 47.95 & 0.58 & 11.7 & 18.0 & 53.2 & 17.1 & 0.00 & وحكو مية فرص للاتصال مع جهات خارجية & 9 \\
\hline 0.00 & 27.70 & 0.49 & 0.00 & 19.8 & 43.2 & 28.0 & 9.0 & الحد من الاخطاء & 10 \\
\hline 0.00 & 17.32 & 0.70 & 36.1 & 27.9 & 27.0 & 9.00 & 0.00 & تخفيضلات التكلفة بتبسيط الإجراءات وتقليل & 11 \\
\hline 0.00 & 37.72 & 0.74 & 40.6 & 33.3 & 24.3 & 1.80 & 0.00 & الأعمال من تأثير العلاقات الثخصية على إنجاز & 12 \\
\hline 0.00 & & & & & & & & تجميع البيانات من مصادر ها الأصلية بصورة & 13 \\
\hline & 21.78 & 0.78 & 45.9 & 41.4 & 712. & 0.00 & 0.00 & موحدة & \\
\hline 500. & .440 & 0.82 & 53.2 & 46.8 & 0.00 & 0.00 & 0.00 & نشر التعليم الالكتروني & 14 \\
\hline 0.01 & 9.24 & 0.76 & 41.5 & 38.7 & 19.8 & 0.00 & 0.00 & خطوة تمهيدية لتنفيذ الحكومة الالكترونية & 15 \\
\hline
\end{tabular}

جدول رقم 3: الفروقات في استجابة افراد العينة حول اهمية تطبيق الادارة الاكترونية

\begin{tabular}{|c|c|c|c|c|c|}
\hline الوظيفة الحالية & مكان العمل & سنوات الخبرة & اللقب العلمي & المؤهل العلمي & العينة \\
\hline 1.327 & 0.28 & 1.31 & 0.874 & 1.554 & $\mathrm{~F}$ \\
\hline 0.258 & 0.258 & 0.266 & 0.501 & 0.204 & Sig. \\
\hline
\end{tabular}




\section{جدول رقم 4: معوقات تطبيق الادارة الاكترونية}

\begin{tabular}{|c|c|c|c|c|c|c|c|c|c|}
\hline الفا & مريع كا & الالالهبية & 5 & 4 & 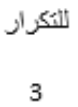 & 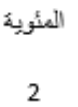 & التشبة & الفترة & ت \\
\hline 0.266 & 2.65 & 0.56 & 10.8 & 28.0 & 28.8 & 25.2 & 7.2 & 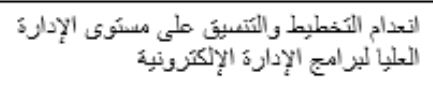 & 1 \\
\hline 0.000 & 43.27 & 0.64 & 27.9 & 20.8 & 30.6 & 19.8 & 0.9 & 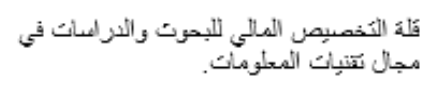 & 2 \\
\hline 0.017 & 8.16 & 0.85 & 73.9 & 21.6 & 4.5 & 0.00 & 0.00 & 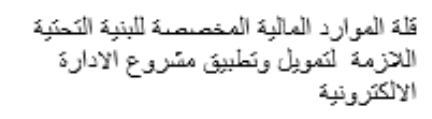 & 3 \\
\hline 0.000 & 28.42 & 0.77 & 62.2 & 14.4 & 13.5 & 7.2 & 2.7 & 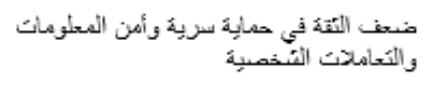 & 4 \\
\hline 0.016 & 5.14 & 0.63 & 0.00 & 61.3 & 27.9 & 8.1 & 2.7 & 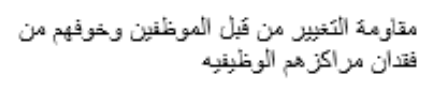 & 6 \\
\hline 0.000 & 32.65 & 0.69 & 31.5 & 36.9 & 15.4 & 16.2 & 0.00 & 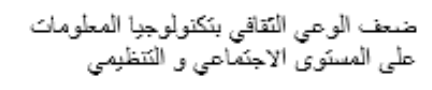 & 8 \\
\hline 0.001 & 15.66 & 0.35 & 2.7 & 8.1 & 13.6 & 32.4 & 43.2 & 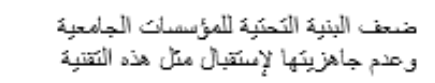 & 11 \\
\hline 0.000 & 16.27 & 0.35 & 2.7 & 8.2 & 13.5 & 32.4 & 43.2 & 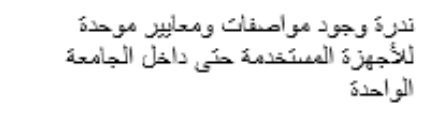 & 12 \\
\hline 0.000 & 19.12 & 0.45 & 0.00 & 18.0 & 28 & 40.5 & 13.5 & الاوضناع الفبائسية والامنية & 14 \\
\hline
\end{tabular}

\section{جدول رقم 5: الفروقات في استجابات افراد العينة حول معوقات تطبيق الادارة الاكترونية}

\begin{tabular}{|c|c|c|c|c|c|}
\hline الوظيفة الحالية & مكان العمل & سنو ات الخبرة & اللقب العلمي & المؤهل العلمي & العينة \\
\hline 2.208 & 0.459 & 1.44 & 1.659 & 0.801 & $\mathrm{~F}$ \\
\hline 0.059 & 0.059 & 0.216 & 0.151 & 0.496 & Sig. \\
\hline
\end{tabular}




\title{
OBSTACLES TO THE APPLICATION OF ELECTRONIC MANAGEMENT IN HIGHER EDUCATION ORGANIZATIONS
}

Samiaah M. Hassen Al-Tmeemy, Wadhah amer Hatem, Kowthrr Hashem, Jasem Kahlef

*sam_saf61@yahoo.com

\begin{abstract}
This study aimed to expose the level of awareness of employees in higher education and scientific research institutions to the importance of applying electronic management in these institutions and the barriers that impede the application (the province of Diyala, case study). Quantitative method is adopted to collect data from Diyala University and Baquba Technical Institute using questionnaire surveys. The sample of (111) was selected using stratified random sampling. Results of the study showed that most of the employees are well aware of the importance of applying the electronic management. The most important obstacle to the adapting electronic management is "the lack of financial resources to finance and implement the project of electronic management". Then followed by "lack of confidence in protecting the confidentiality and security of information ", and" the weakness of the cultural awareness of information technology at social and organizational level ". In the light of the results of the study, several recommendations were presented; including necessity of providing financial support to apply electronic management and attention to infrastructure for the higher education system to keep up with development that is happening in the context of technology. Also, it is necessary to provide preventive security to ensure the protection of data from breakthrough.
\end{abstract}

